\title{
Announcement: Physical Review $C$ invites milestone research on nuclear instrumentation
}

Physical Review $C$ currently publishes new experimental approaches and techniques only as part of a paper that is also reporting significant nuclear physics research results. However, milestone experimental developments take time before they produce important experimental results. Therefore, Physical Review $C$ is expanding its scope to include papers that report significant advances in instrumentation for nuclear science, even when new measurements are not yet available.

Manuscripts focused on instrumentation should be relevant to major progress in nuclear science research and demonstrate a strong connection to advances in nuclear physics in order to be considered for publication. They must be physics-oriented and open new opportunities. Instrumentation papers will appear in the appropriate subject matter section of PRC.

We hope that readers will find it useful to learn about important instrumentation developments while important physics results from these instruments are beginning to take shape.

Benjamin F. Gibson

Editor

Physical Review C

(Q) Published 21 July 2021

DOI: 10.1103/PhysRevC.104.010001 